\title{
Swing analysis by body type with golf shot analysing device
}

\section{Jaeyoung Park}

Department of Exercise Prescription,

Dongshin University,

Daeho-dong, Naju-si,

Jeollanam-do, 58245, Republic of Korea

Email: koungjun1@nate.com

\section{Minwoo Cheon}

Department of Health Administration,

Dongshin University,

Daeho-dong, Naju-si,

Jeollanam-do, 58245, Republic of Korea

Email: mwcheon@dsu.ac.kr

\section{Jongjin Bae}

Jeollanamdo Sports Council,

Samhyang-eup, Muan-gun,

Jeollanam-do, 58565, Republic of Korea

Email: bjj1008@hanmail.net

\section{Jungchul Lee*}

Department of Exercise Prescription,

Dongshin University,

Daeho-dong, Naju-si,

Jeollanam-do, 58245, Republic of Korea

Email: channel365@hanmail.net

${ }^{*}$ Corresponding author

\begin{abstract}
This study is to provide fundamental resources to develop scientific golf swing method by analysing golf shot change by swing patterns for three different body types. Swing analysis was performed by using FlightScope ${ }^{\circledR}$ golf shot analysing device on three different body types with proper swing methods. Driving distance, driving direction, and head speed were analysed in this way. The result showed a correlation between the groups and swing patterns in terms of driving distance, as well as positive relation between the groups and swing patterns. In terms of driving direction and head speed, there was correlation between the groups, swing patterns and the difference was shown between swing patterns. Therefore, it was proved that different swing patterns need to
\end{abstract}


be applied for each body type. Further study needs to be conducted using FlightScope $^{\circledR}$ for scientific assessment. For the enhanced interoperability between sports science and IT technology, convergence education system models should be also continuously developed.

Keywords: FlightScope ${ }^{\circledR}$; golf swing; ectoderm; endoderm; mesoderm; convergence education systems; IT technology.

Reference to this paper should be made as follows: Park, J., Cheon, M., Bae, J. and Lee, J. (2016) 'Swing analysis by body type with golf shot analysing device', Int. J. Advanced Media and Communication, Vol. 6, No. 1, pp.39-47.

Biographical notes: Jaeyoung Park received his $\mathrm{MS}$ and $\mathrm{PhD}$ in the Department of Sports Biomechanics from Chonnam National University in 2004 and 2008, respectively. He is a Professor of Exercise Prescription Department of current Dongshin University. His current research interests are gait analysis, exercise motion analysis, corrective exercise device and development of vertebral column proofreading device.

Minwoo Cheon received his BS, MS and PhD in the Department of Electrical and Electronic Engineering from Dongsin University in 2001, 2003 and 2006, respectively. Also, he received his $\mathrm{PhD}$ in College of Medicine from Chosun University in 2008. Since 2010, he has been with the Department of Biomedical Engineering, the Department of Health Administration, Dongshin University as an Assistant Professor. He was awarded a Paper and a Scholarship at KIEEME in 2007, 2008 and 2013, respectively. His current research interests are bio artificial organs, light therapy, rehabilitative medicine and developmental medicine.

Jongjin Bae was granted a Doctorate in the Department of Physical Education from Korea University in 2004. He was a Professor at Hannam \& Dongshin University and GongJu National University of Education from 2005 to 2013. Since 2014, he is doing exercise consulting for elite athletes in Sports Science Center of Jeollanamdo sports council. His current research interests are sports entertainment and IT for sports.

Jungchul Lee received his $\mathrm{MS}$ and $\mathrm{PhD}$ in the Department of Physical Education from Korea University in 2002 and 2006, respectively. He was a Exercise Specialist at Seoul Asan Medical Center from 1999 to 2000. He is currently being served as a Professor in the Department of Exercise Prescription from the Dong-Shin University. His current research interests are exercise prescription, exercise rehabilitation, sports medicine and scientific program development of Nordic working.

This paper is a revised and expanded version of a paper entitled 'Golf tee analysis per body type utilizing FlightScope $\mathrm{X} 1^{\circledR}$, presented at 2015 International Conference on Platform Technology and Service (PlatCon-15), Jaeyoung Park, Jeju, Korea, 26 January, 2015. 


\section{Introduction}

Golf swing is connected like a chain so it delivers powerful momentum to the ball upon impact and gets club head exercised by delivering kinetic energy to the ball as much as possible with proper arrangement of the body (Richards et al., 1985). It is also very important to have accuracy of the impact and rhythmically coordinated movement of the club head to move the ball towards designated direction after the impact (Milburm, 1982). To practice ideal swing and accurate driving distance, it is very important to reveal the kinematical characteristics of shot with proper swing method for body type. Achieving one's own swing method for our body and physical ability is critical to obtain the swing with power and consistency.

Everyone has a different body type and shape with different physical abilities; therefore, applying standardised swing to different people with different body types is not easy, and improvement rate also gets decreased (Adams et al., 1998). In particular, copying the swing movement of someone with significantly better ability and conditions like a professional golfer can cause serious sports injury through excessive swing movements; it can also decrease exercise performance ability. For this reason, it is required to analyse the average data of head speed simultaneously with swing analysis, height and direction of the ball to obtain the right swing method.

Presently, devices combining sports and IT technology have been presented along with software customised to each event. Given the nature of golf, a multi-angled approach in $3 \mathrm{D}$ space is more effective in analysis. It is also effective to perform the analysis in the three-dimensional space with multi-lateral approach and three-dimensional analysing system such as FlightScope $3 \mathrm{D}$ analysis system (FlightScope ${ }^{\circledR}$ EDH Inc, South Africa), which is utilised to get the accurate swing method by individual body type.

Therefore, this study targeted three representing body types to provide fundamental resources of ideal golf swing method by analysing golf shot with systematic swing patterns for each body type. Also, in this research, the convergence education system for improving interoperability in sports science and technology is presented.

\section{Experiment}

\subsection{Subject of study}

Golf players with less than three years of experience were targeted and three groups (endoderm, mesoderm and ectoderm) of six players which made 18 in total were selected for this study. Physical characteristics of the participants are shown in Table 1.

\subsection{Way of measuring and process}

This study is to find out the influence of swing pattern by body type on driving distance, driving direction and head speed. Test valve and equipment are shown in Table 2. 
Table 1 Mean and SD of physical characteristics $(n=18)$

\begin{tabular}{lcccccc}
\hline Group & Age $(\mathrm{yr})$ & Height $(\mathrm{cm})$ & Weight $(\mathrm{kg})$ & Chest $(\mathrm{cm})$ & Carrier $(\mathrm{yr})$ & Handy \\
\hline Endoderm (6) & 20.00 & 174.67 & 83.00 & 106.67 & 2.17 & 8.33 \\
& \pm 1.00 & \pm 2.52 & \pm 1.00 & \pm 2.89 & \pm 0.29 & \pm 1.53 \\
Mesoderm (6) & 19.66 & 179.00 & 68.00 & 96.67 & 2.27 & 8.33 \\
& \pm 1.52 & \pm 3.00 & \pm 2.00 & \pm 2.89 & \pm 0.64 & \pm 2.52 \\
Ectoderm (6) & 19.33 & 179.00 & 73.33 & 101.67 & 2.00 & 7.67 \\
& \pm 1.45 & \pm 3.00 & \pm 1.53 & \pm 2.89 & \pm 0.54 & \pm 0.58 \\
\hline
\end{tabular}

Table 2 List of measurement

\begin{tabular}{ll}
\hline List & Inspection equipment \\
\hline Weight & In-body (BIOSPACE, Republic of Korea) \\
Height & In-body (BIOSPACE, Republic of Korea) \\
Driving distance & FlightScope $^{\circledR}$ (EDH Inc, South Africa) \\
Driving direction & FlightScope $^{\circledR}$ (EDH Inc, South Africa) \\
Ball speed & FlightScope $^{\circledR}$ (EDH Inc, South Africa) \\
\hline
\end{tabular}

The main function of the program utilised in this study is to change the swing data into kinetic variable. As shown in Figure 1, Analysis of this study was the measurement system based on the 3D Doppler tracking ball. This device measures the variables related to the ball, the club or the swing pattern through the golf swing.

A test procedure of this study is shown in Figure 2.

Figure 1 Analysis of golf swing variables (see online version for colours)
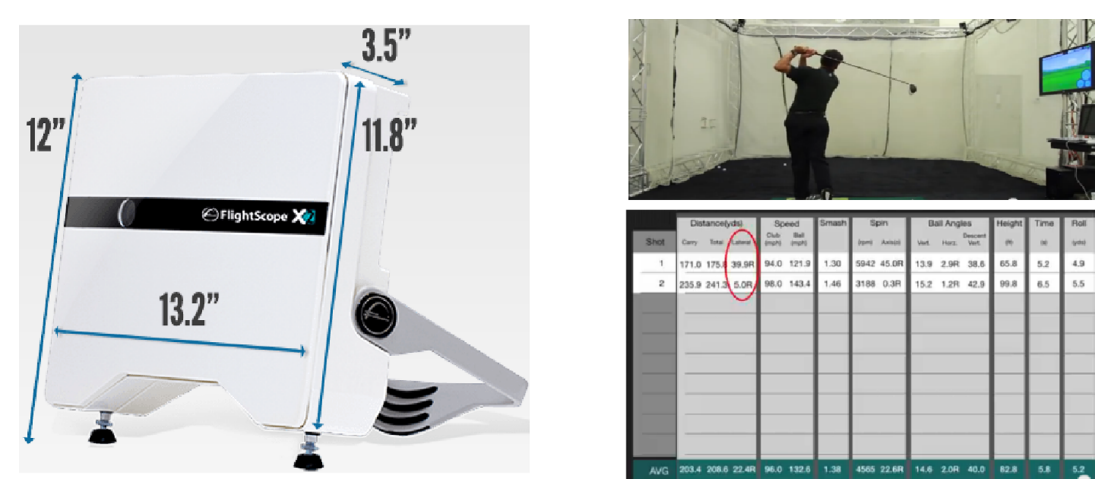

\subsection{Data processing method}

According to the data processing utilised in this study, measured data are stored in the database; then, the average and standard deviation are calculated by measurement item using SAS Windows 9.2 statistics package. Also, the average and standard deviation by group and by pattern were calculated. In addition, two-way ANOVA was performed to show the correlation between the groups and pattern. Statistical significance level was set as $P<0.05$. 
Figure 2 Inspection processes and procedures

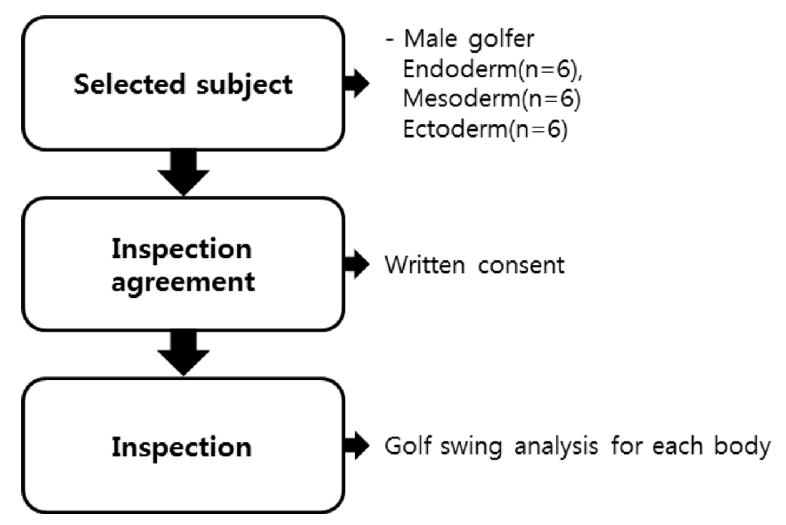

\section{Results and discussion}

The results from analysing the influence of swing patterns by body type on driving distance, driving direction and speed of ball are as below.

\subsection{Driving distance}

Changes in driving distance by using shot analysing device are shown in Table 3.

The longest driving distance was shown when the ectoderm group used the swing pattern for the ectoderm body type. Same results were presented for the endoderm group as well as the mesoderm group.

As shown in Table 4, the result from multi-variate analysis indicated a correlation $(F=12.8, P<0.0001)$, and there was positive relation between the groups $(F=3.27$, $P<0.0408)$ and patterns $(F=7.04, P<0.0012)$. In other words, driving distance varied depending on the groups and patterns.

Table 3 Mean and SD of driving distance in each group (m)

\begin{tabular}{lccc}
\hline Group & Pattern- $A$ & Pattern- $B$ & Pattern-C \\
\hline A & $231.50 \pm 2.46$ & $226.96 \pm 2.23$ & $229.31 \pm 3.27$ \\
B & $227.60 \pm 5.34$ & $233.25 \pm 3.81$ & $230.58 \pm 4.69$ \\
C & $227.08 \pm 4.88$ & $232.13 \pm 2.50$ & $234.00 \pm 3.21$ \\
\hline
\end{tabular}

A: Endoderm; B: Mesoderm; C: Ectoderm.

Table 4 The result of two-way ANOVA about driving distance

\begin{tabular}{lcrrrr}
\hline Source & $D F$ & $S S$ & $M S$ & $F$ & $P$ \\
\hline Group & 2 & 92.57 & 46.28 & 3.27 & 0.0408 \\
Pattern & 2 & 199.64 & 99.82 & 7.04 & 0.0012 \\
Group $\times$ Pattern & 4 & 733.38 & 183.34 & 12.94 & $<0.0001$ \\
\hline
\end{tabular}

DF: Degree of freedom; SS: Sum of squares; Mean squares: SS/DF; F: F-value; P: $P$-value. 


\subsection{Driving direction}

Changes in shot by using shot analysing device are shown in Table 5. The most accurate driving direction was presented when the ectoderm group used the swing pattern for the ectoderm body type. Same results were presented for both the endoderm group and the mesoderm group. The most accurate driving directions were shown when the group used the pattern suited for their body types.

Table 5 Mean and SD of angle in each group (degree)

\begin{tabular}{lrcc}
\hline Group & Pattern- $A$ & Pattern- $B$ & Pattern-C \\
\hline A & $-0.16 \pm 0.51$ & $0.61 \pm 0.84$ & $-0.72 \pm 0.82$ \\
B & $0.11 \pm 0.90$ & $0.27 \pm 0.46$ & $-0.77 \pm 0.64$ \\
C & $0.33 \pm 0.90$ & $-0.55 \pm 0.70$ & $0.22 \pm 0.54$ \\
\hline
\end{tabular}

A: Endoderm; B: Mesoderm; C: Ectoderm.

As shown in Table 6 , the result from multivariate analysis revealed a correlation $(F=12.43, P<0.0001)$, and negative relation was shown between the groups $(F=0.46$, $P<0.6335)$.

Table 6 The result of two-way ANOVA about ball angle

\begin{tabular}{lccccc}
\hline Source & $D F$ & $S S$ & $M S$ & $F$ & \multicolumn{1}{c}{$P$} \\
\hline Group & 2 & 0.48 & 0.24 & 0.46 & 0.6335 \\
Pattern & 2 & 10.03 & 5.01 & 9.54 & 0.0001 \\
Group $\times$ Pattern & 4 & 26.14 & 6.53 & 12.43 & $<0.0001$ \\
\hline
\end{tabular}

DF: Degree of freedom; SS: Sum of squares; Mean squares: SS/DF; F: F-value; P: $P$-value.

While positive relation was shown between the patterns $(F=9.54, P<0.0001)$. In other words, driving distance varied depending on the swing patterns.

\subsection{Head speed}

Analysed result of speed using shot analysing device is shown in Table 7.

Table 7 Mean and SD of head speed in each group $(\mathrm{m} / \mathrm{s})$

\begin{tabular}{lccc}
\hline Group & Pattern- $A$ & Pattern- $B$ & Pattern-C \\
\hline A & $32.37 \pm 1.78$ & $30.60 \pm 2.18$ & $31.22 \pm 2.03$ \\
B & $31.36 \pm 2.31$ & $32.89 \pm 3.57$ & $31.66 \pm 2.84$ \\
C & $29.78 \pm 2.05$ & $31.67 \pm 2.16$ & $33.47 \pm 2.25$ \\
\hline
\end{tabular}

A: Endoderm; B: Mesoderm; C: Ectoderm. 
The fasted speed was detected when the ectoderm group used the pattern for the ectoderm body type. Same results were detected for both the endoderm group and the mesoderm group. The fastest speeds were shown when the group used the pattern for their body types.

As shown in Table 8, result from multi-variate analysis showed a correlation $(F=6.50, P<0.0001)$, and negative relation was shown between the groups $(F=0.77$, $P<0.4645)$.

Table 8 The result of two-way ANOVA about head speed

\begin{tabular}{lcrccc}
\hline Source & $D F$ & $S S$ & $M S$ & $F$ & $P r$ \\
\hline Group & 2 & 8.95 & 4.47 & 0.77 & 0.4645 \\
Pattern & 2 & 24.37 & 12.18 & 2.10 & 0.1264 \\
Group $\times$ Pattern & 4 & 151.12 & 37.78 & 6.50 & $<.0001$ \\
\hline
\end{tabular}

DF: Degree of freedom; SS: Sum of squares; Mean squares: SS/DF; F: F-value; P: $P$-value.

While positive relation was shown between the patterns $(F=7.04, P<0.0012)$. This means that head speed varied depending on the swing patterns.

In this research, a convergence education model between golf and IT system was suggested based on FlightScope ${ }^{\circledR}$ 3D analysis system by examining body type-specific golf swings and maximising system standardisation and interoperability. The idea of the suggested model is a component model combining sports and computer program technology convergence education systems and e-learning. The objective of this study is to find out the right swing pattern for three different body types: ectoderm, mesoderm and endoderm. For this purpose, six participants for each body type were selected for a total of 18 participants, and driving distance, driving direction and head speed were analysed by comparing three different swing patterns with FlightScope ${ }^{\circledR}$ golf shot analysing device.

It is said that the maximum power and ideal swing can be achieved by doing a powerful swing onto the ground using a leg and ground will push the body of golfer with the same power against it. This ground reaction force is eventually delivered to the golf club and ball passing through the leg, pelvis and both arms (Patria et al., 2005).

Using external force is a general way to improve the stabilisation of the lower body and rotation axis as well as driving distance (Gluck et al., 2008). However, pushing the ball away by accelerating the club head upon impact is a physically proven way. Therefore, the factor for individual's ideal swing needs to be reviewed apart from the swing methods using ground reaction force.

There have been many attempts in preceding research to find the driving distance by swing mechanism and its relationship with body performance (Hay, 1993; Nagano and Sawada, 1974; Smith, 1998; Williams, 1967).

Also, analyses of the decision factors (Broer, 1973; Campbell and Reid, 1985) of driving distance upon golf swing were performed. Williams (1967) performed an analysis of the direction of the club head during backswing by professional players, and the results varied depending on the player's body type. 
On the basis of these preceding researches, this study revealed that the maximum results can be achieved when using the right swing pattern suited for the somato type in terms of driving distance, driving direction and head speed. Sports science is about finding the optimal model by using accumulated measurements. In other words, the position of technical movement can be measured through scientific device which can capture the momentary movement. Golf is a scientific exercise that requires accurate exercise skill and high tech device. Successful outcome can be achieved by having the right performance ability for the individual. It is expected that continued convergence study will facilitate the development of an optimal sports convergence education model for sports performance improvement based on IT equipment.

\section{Conclusions}

Swing analysis by body type with golf shot analysing device. This study is to provide fundamental resources to develop scientific golf swing method by analysing golf shot change by swing patterns for three different body types.

According to the analysis of the swing patterns for each body type, correlation was shown between the patterns and body types; positive relation was also shown between the swing patterns and body types in terms of driving distance. For driving direction, correlation was shown between the swing patterns and body types, and positive relation was also shown between them. In terms of head speed, correlation was also shown between the swing patterns and body types, and positive relation was also shown between them.

The above results indicate that the ideal swing pattern can be achieved when using the right swing pattern suited for each body type. Furthermore, it is also suggested that systematic design and preparation need to be performed using scientific device to measure and analyse the most effective swing methods.

\section{References}

Adams, M., Tomasi, T.J. and Suttie, J. (1998) The Laws of the Golf Swing, Harper Collins Publishers, New York.

Broer, M.R. (1973) The Efficiency of Human Movement, 3rd ed., W.B. Saunders Co., Philadelphia, pp.224-248.

Campbell, K. and Reid, R. (1985) 'The application of control theory to simplified models of complex human motions: the golf swing', in Winter, D., Norman, R., Wells, E., Hayes, K. and Patla, A. (Eds.): Biomechanics $I X-B$, Baltimore Human Kinetics, Champaign, Illinois, pp.527-538.

Gluck, G.S., Bendo, J.A. and Spivak, J.M. (2008) 'The lumber spine and low back pain in golf: a literature review of swing biomechanics and injury prevention', The Spime Journal, Vol. 8, pp.778-788.

Hay, J.G. (1993) The Biomechanics of Sports Techniques, 4th ed., Prentice Hall, New Jersey.

Milburm, P. (1982) 'Summation of segmental velocities in the golf swing', Medicine and Science in Sports and Exercise, Vol. 14, No. 1, pp.60-64.

Nagano, N. and Sawada, Y. (1974) 'A kinematic analysis of the golf swing by means of fast motion picture in connection with racial difference', Journal of Sports Medicine and Physical Fitness, Vol. 14, pp.55-63. 
Patria, A.H., Justin, K. and Duncan, R. (2005) 'The role of biomechanics in maximising distance and accuracy of golf shots', Sports Med., Vol. 35, No. 5, pp.429-449.

Richards, J., Farrell, M., Kent, J. and Kraft, R. (1985) 'Weight transfer pattern during the golf swing', Research Quarterly for Exercise and Sports, Vol. 56, No. 4, pp.365-365.

Smith, R. (1998) How to Find Your Perfect Golf Swing, Broadway Books, New York, NY.

Williams, D. (1967) 'The dynamics of the golf swing', Quarterly Journal of Mechanics and Applied Mathematics, Vol. 20, pp.247-264. 